\title{
Treatment results of curative gastric resection from a specialist Australian unit: low volume with satisfactory outcomes
}

\author{
Iain G. Thomson · David C. Gotley • \\ Andrew P. Barbour • Ian Martin • Neil Jayasuria • \\ Janine Thomas $\cdot$ Bernard M. Smithers
}

Received: 29 August 2012 / Accepted: 3 February 2013/Published online: 9 March 2013

(c) The International Gastric Cancer Association and The Japanese Gastric Cancer Association 2013

\begin{abstract}
Background The incidence of gastric cancer is decreasing in Australia, yet it remains a common cause of cancerrelated mortality. Surgical resection remains the cornerstone of curative treatment. High-volume specialized units have reported superior perioperative and oncological outcomes. The role of D2 lymphadenectomy has been controversial as a result of concerns over increased morbidity. Our aim is to report the perioperative and oncological outcomes of curative gastric resection from a specialist Australian upper GI unit.

Methods Data from a prospectively maintained database were reviewed for all patients undergoing curative resection for gastric adenocarcinoma from a single unit during a 12-year period. Perioperative and long-term outcomes were compiled.

Results There were 255 curative gastric resections during 12 years. An R0 resection was performed in $96 \%$ with a perioperative mortality rate of $1.6 \%$. A D2 dissection was performed in $85 \%$ of cases in the past 6 years, with no
\end{abstract}

Electronic supplementary material The online version of this article (doi:10.1007/s10120-013-0240-3) contains supplementary material, which is available to authorized users.

I. G. Thomson - D. C. Gotley · A. P. Barbour · I. Martin ·

N. Jayasuria - J. Thomas - B. M. Smithers

Upper Gastrointestinal and Soft Tissue Unit, Princess Alexandra

Hospital, Brisbane, QLD, Australia

I. G. Thomson $(\bowtie) \cdot$ D. C. Gotley · A. P. Barbour .

B. M. Smithers

Department of Surgery, The University of Queensland,

Princess Alexandra Hospital, Ipswich Road, Woolloongabba,

Brisbane, QLD 4102, Australia

e-mail: i.thomson@uq.edu.au increase in perioperative morbidity or mortality detected. The 5-year overall survival was $53 \%$.

Conclusion Our results demonstrate that both short- and long-term outcomes of surgical resection in gastric cancer patients, comparable to international high-volume centers, can be achieved in an Australian upper GI unit. A D2 lymph node dissection can be performed safely without any increase in perioperative risk in a specialist unit that has the necessary training but also the perioperative support structures to manage these complex patients.

Keywords Gastric cancer - Gastrectomy .

Surgical volume

\section{Introduction}

Despite a reduction in incidence, gastric cancer remains a significant worldwide problem, being the second leading cause of cancer-related mortality worldwide [1,2]. Within Australia the incidence of gastric cancer has decreased; it is now the 12th most common malignancy. A recent review of cancer in Australia demonstrated that gastric cancer has the fifth lowest survival rate of cancers in Australia. Therefore despite a reduced incidence it remains an important cause of cancer-related mortality [3].

The role of surgery for gastric cancer remains an integral aspect of any curative approach, but the extent of lymph node dissection in gastric cancer is still being debated in Western countries. Nodal status is a key prognostic factor [4]. A more aggressive lymphadenectomy allows for more accurate staging [5], as recognized in the AJCC staging manual (seventh edition), which states at least 16 nodes need to be examined for adequate nodal staging [6]. Extended lymphadenectomy (D2/D3) for 
gastric cancer is routinely performed in Japanese surgical units $[7,8]$, with large reported series demonstrating that it can be performed safely $[9,10]$. Two European randomized studies assessing the role of extended lymph node dissection (D2) failed to show any improvement in survival $[11,12]$, but with associated higher mortality and morbidity rates in the extended lymphadenectomy group, as a result of splenic and pancreatic resection. This result has led to a shift from routine resection of the distal pancreas and spleen to a much more selective approach [13-16]. A more recent randomized study from a Western surgical center was able to demonstrate no increase in morbidity or mortality rates with D2 lymphadenectomy [17]; this has also been reported in numerous nonrandomized studies from specialized units with an interest in gastric cancer, demonstrating that a D2 lymph node dissection can be performed safely with good outcomes [10, 15, 18-21].

The management of gastric cancer has progressed during the past decade with the addition of multimodal therapy and a multidisciplinary approach. The use of neo-adjuvant chemotherapy based on the results of the MAGIC trial in those patients with locally advanced gastric cancer [22] has been a major positive development during the past decade. This approach is widely utilized in Australia, UK, and Europe. The use of adjuvant chemoradiation based on the INT-0116 trial [23] is still widely employed in the United States. In Japan and parts of Asia, adjuvant chemotherapy in the form of S1 is used [24]. More recently adjuvant chemotherapy has been shown to be beneficial in a large study from Korea [25]. All these methods increase the complexity of managing patients with gastric cancer and require a multidisciplinary team (MDT) input to achieve the best possible outcome for the patient.

There is evidence linking improved outcomes for patients with increasing surgical volume, with the largest difference seen in esophageal or pancreatic resections [26]. There are also data demonstrating improved outcomes with increasing surgical volume for gastric resection. Data from the United States have demonstrated that hospitals that perform fewer than five gastric resections annually had a mortality of $13 \%$ compared with $8.7 \%$ in high-volume hospitals [27]. A cohort study investigating esophagogastric cancer management in England demonstrated that surgical volume of both the surgeon and the hospital was inversely related to mortality [28]. A systematic review of studies investigating volume and mortality in gastrointestinal cancer found that volume is inversely related to mortality across all subtypes, including gastric cancer [29]. For these and other reasons, gastric cancer services have been centralized in some countries.

There is a paucity of data relating to surgical outcomes in gastric cancer from surgical units in Australia, which all tend to manage low volumes of the disease given the low incidence in this country. We have evaluated the surgical outcomes from patients undergoing resection for gastric cancer, in an upper gastrointestinal unit, in Australia, during a 12-year period. The aim was to examine the shortterm perioperative morbidity and mortality in those undergoing curative surgery, including those undergoing a D2 lymphadenectomy. Secondary aims were to examine any changes in management over this period and compare these results of our perioperative outcomes and cancer outcomes to those published in the literature.

\section{Materials and methods}

\section{Patients}

A prospective database of all patients with gastric cancer presenting to the Princess Alexandra Hospital Upper Gastrointestinal Unit has been maintained from 2005, with retrospective accrual of patients managed back to 1993 . The hospital ethics committee approved this clinical database. For this study we have examined a consecutive cohort of patients treated in our unit from January 2000 to December 2011. Patients were staged preoperatively with an endoscopy and computed tomography (CT) of the chest, abdomen, and pelvis. Diagnostic laparoscopy and peritoneal washings have been used selectively for patients requiring a total gastrectomy and those with apparent T3 tumors. Endoscopic ultrasound has also been employed selectively during this period as it became available in our institution. Demographic, pathological, and treatmentrelated variables were recorded prospectively into the database. Of the 518 patients in the cohort, 167 (32\%) were treated with palliative intent. The remaining 351 were treated with curative intent. We have excluded nonadenocarcinoma pathology, in situ disease, those undergoing endoscopic or nonstandard resection, patients who refused treatment, and those who progressed on treatment or who were unresectable. This selection resulted in 255 study patients with gastric adenocarcinoma who were treated with curative intent undergoing either a total or subtotal gastrectomy (see Appendix 1 in ESM for exclusion details).

\section{Surgery}

One of four consultant surgeons in the Princess Alexandra Upper Gastrointestinal Surgery Unit was responsible for each of the resections. The type of resection was based on tumor location with the aim for a R0 resection. Subtotal gastrectomy was performed for distal or antral cancers where a $5-\mathrm{cm}$ proximal margin could be easily attained. Total gastrectomy was performed for proximal cancers and 
cancers involving the body of the stomach where a $5-\mathrm{cm}$ proximal margin was not possible with a subtotal resection. The extent of lymphadenectomy was tailored to the individual patient and was defined using the Japanese gastric cancer treatment guidelines classification [30]. A D1 gastrectomy was defined as the appropriate removal of the primary plus the first level of draining nodes. A D1+ resection implies the same with removal of some but not all the appropriate second-level nodes, and a D2 resection implies the removal of all the first and second level of draining lymph nodes appropriate for a tumor at a particular primary site in the stomach.

Patients without comorbidities, and those less than 80 years of age with a tumor considered to be T2 or more, were considered for a D2 resection. A lesser lymphadenectomy, either a D1+ or D1 resection, was usually performed in patients with substantial medical comorbidities, those aged over 80 or with early $\mathrm{T}$ stage lesions $(<\mathrm{T} 2)$. As our experience with $\mathrm{D} 2$ resection has increased, we have become more aggressive in its utilization. When the evidence suggested it was appropriate, all patients considered fit for preoperative chemotherapy with tumors $>\mathrm{T} 2$ had a D2 lymph node dissection. Distal pancreatectomy and splenectomy were only performed in cases where there was suspicion of direct invasion or obvious likely involvement of the station 10 nodes, for example, a fundal primary tumor.

More recently, laparoscopic approaches have been utilized initially for patients with early-stage disease or the elderly with localized primary lesions considered not suitable for aggressive lymphadenectomy. A D2 resection has been performed laparoscopically in highly selected patients, notably those who are thin and have small primary cancers.

Pathological analysis

On most occasions the resected specimens were prepared in the operating theater by a member of the surgical team dissecting the nodal tissue into separately labeled containers. Care was taken not to disturb the tissue margins at the site of the primary lesion. The specimens were evaluated by routine hematoxylin and eosin staining. Tumor stage was assessed by experienced gastrointestinal pathologists and classified according to the seventh edition of the TNM staging system of the American Joint Committee on Cancer for gastric cancer [6]. Positive proximal or distal margins were defined as microscopic tumor seen at the duodenal or gastroesophageal transection margin.

Perioperative systemic therapy

Before the report of the MAGIC trial, patients with extensive nodal metastasis were considered for postoperative chemoradiation as per the Intergroup trial protocol
[23]. With reports suggesting a resection with extensive lymphadenectomy (D2) negating the effect of postoperative chemoradiation [23], we moved toward a more aggressive surgical approach (D2) and away from postoperative therapy. Following the published outcomes from the MAGIC trial [22], we have routinely offered perioperative chemotherapy as per the trial protocol, to patients with tumours that are $\mathrm{T} 2$ or greater and who are considered fit to receive chemotherapy.

\section{Complications and follow-up}

Procedure-related mortality was defined as any death during the hospital stay or within 30 days of surgery. All unexpected postoperative events were recorded as morbidity. Respiratory complications were defined as suspicion of chest infection associated with inflammatory response with or without radiologic or microbiological confirmation for which active treatment was offered. Cardiac morbidity was defined as any event requiring input from the cardiology service, such as myocardial infarction, arrhythmia, or acute congestive cardiac failure. Anastomotic leak was considered as any evidence of a defect in the anastomosis: this was defined as clinically significant on the basis of any intervention that was required because of the patient's condition, and radiologic if a defect was reported on a contrast swallow study and if there was no evidence of clinical sequelae. Following discharge, patients were assessed every 3 months for the first 2 years, then every 6 months for the next 3 years, and then annually or as required. When assessed at these time points, a history and physical examination was performed with investigations directed toward any new symptoms or signs.

Statistical analysis

Statistical analysis was carried out with SPSS for Windows version 20. To examine for any change in management during the 12-year period, the cohort was divided into 6-year groups. Overall survival rates were calculated using the Kaplan-Meier method. The chi-square or Fisher exact test was used for comparison of categorical variables; the Mann-Whitney $U$ test was used to compare median values for continuous variables. Comparison of survival was performed with the log-rank test (Mantel-Cox) test.

\section{Results}

Patients

There were 255 patients identified who underwent a curative total or subtotal gastrectomy at the PAH during the 
12-year period. The median age was 69 , with a 3:2 male dominance. The tumor involved the proximal stomach in $37 \%$, mid-stomach in $36 \%$, and distal stomach in $24 \%$ of the cases.

\section{Staging and treatment overview}

All 255 patients (100\%) had a CT scan of the abdomen/ pelvis as part of their staging investigations. Diagnostic laparoscopy was employed in only $102(40 \%)$ of patients, and endoscopic ultrasound (EUS) was utilized in $26(10 \%)$ of patients. Following staging investigations, 202 patients (79\%) proceeded directly to surgery; $45(17 \%)$ patients underwent neoadjuvant chemotherapy. The use of postoperative adjuvant chemoradiotherapy was utilized in only 8 patients $(3 \%)$.

\section{Pathological features}

The postoperative stage of patients in this series is shown in Fig. 1, with 83 (33\%) patients being stage III or above. The cohort has $127(49 \%)$ cases of T3 or T4 lesions and $140(55 \%)$ cases with positive lymph nodes. There were no significant differences in the $\mathrm{T}, \mathrm{N}, \mathrm{M}$, or overall stage groupings across the two time periods.

\section{Surgery and perioperative outcomes}

Of the 255 patients who underwent resection, $21(8 \%)$ had a laparoscopic approach: 132 total gastrectomies (52\%) and 123 subtotal gastrectomies ( $48 \%$ ) were performed. An R0 resection was performed in 245 cases (96\%), and 10 cases $(4 \%)$ had microscopic positive margins. The median nodal count was 21 for the entire cohort. The percentage of patients with greater than 15 lymph nodes examined was

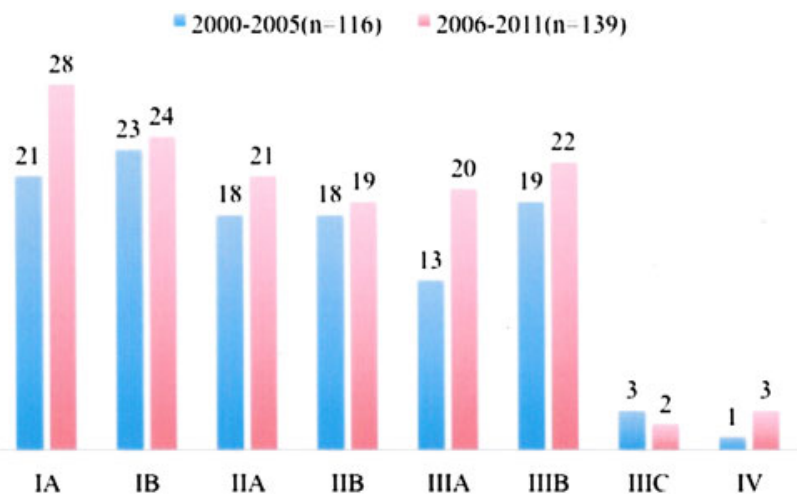

Fig. 1 Pathological and postoperative stage grouping. Overall stage (AJCC 7th edition)
$67 \%$. A D2 lymph node dissection was performed in 146 cases $(57 \%)$. Other organs were resected on 13 occasions (spleen 11, distal pancreas 1, colon 1). Operative mortality occurred in $4(1.6 \%)$ patients: the cause was pulmonary embolism in 2 patients on postoperative day 3 and 5 , myocardial infarction on postoperative day 5 in 1 patient, and aspiration and respiratory arrest on postoperative day 13 in 1 patient. Complications occurred in 99 patients (39\%) (Table 2). The majority of morbid events were related to respiratory or cardiac morbidity. An anastomotic leak was identified in 23 patients $(9 \%)$. Following a total gastrectomy, 19 patients (14\%) demonstrated a leak at the esophagojejunal anastomosis, and following a subtotal gastrectomy, 4 patients $(3 \%)$ demonstrated a leak. In this group, the leak occurred at the anastomosis in 2 cases and at the duodenal stump in 2 cases.

\section{Follow-up and survival}

The median follow-up for surviving patients was 46 months (range 0-137 months). There have been 107 deaths during the follow-up period with 148 patients remaining alive at the last follow-up. There have been 100 patients with recurrent disease, 4 of whom are still alive with recurrent disease. The 5-year overall survival for all patients was $53.1 \%$; the 5-year disease-free survival for all patients was $54.3 \%$.

Management differences during 6-year periods

The two most significant differences in the management of patients during the two time periods was a significant increase in the use of perioperative neoadjuvant chemotherapy, which has increased from 9 cases $(8 \%)$ to 36 cases $(26 \%)$ in the period from 2006 until the present ( $p \leq 0.001$; Table 1) and the increased use of $\mathrm{D} 2$ resection, rising from 30 cases $(26 \%)$ to 116 (84\%) cases $(p \leq 0.001$; Table 2) As a result of this increased nodal dissection, the median nodal count has increased from 16 to $24(p=0.001)$ across the two time periods. This span has also seen the percentage of patients with more than 15 lymph nodes examined rise from $52 \%$ in the period from 2000-2005 to $86 \%$ in the period 2006-2011. Despite the increased use of chemotherapy and more extensive lymph node dissection there has been no significant increase in overall morbidity, with an increase in cardiac complications since 2006 being the only significant difference between the two time periods (Table 2).

\section{D1 compared to D2 resection}

We have analyzed the cases for any differences with regard to treatment plan, surgical approach, and short- and long- 
Table 1 Patient demographics, staging investigation, and treatment overview of 255 curative gastric resections

\begin{tabular}{|c|c|c|c|c|}
\hline & All & 2000-2005 & 2006-2011 & $p$ value \\
\hline Number of patients & 255 & 116 & 139 & \\
\hline Age (years) & $69(16-89)$ & $69(26-89)$ & $68(16-89)$ & n.s \\
\hline Sex (M:F) & $3: 2$ & $3: 2$ & $3: 2$ & n.s \\
\hline Body mass index (BMI) & $26(15-47)$ & $25(16-43)$ & $27(15-47)$ & n.s \\
\hline Tumor site & & & & 0.012 \\
\hline Upper & $94(37 \%)$ & $42(36 \%)$ & $52(37 \%)$ & \\
\hline Mid & $92(36 \%)$ & $52(45 \%)$ & $40(28 \%)$ & \\
\hline Lower & $60(24 \%)$ & $21(18 \%)$ & $39(28 \%)$ & \\
\hline Whole & $9(4 \%)$ & $1(1 \%)$ & $8(6 \%)$ & \\
\hline ASA & & & & n.s \\
\hline 1 & $6(2 \%)$ & $4(3 \%)$ & $2(1 \%)$ & \\
\hline 2 & $173(68 \%)$ & $76(66 \%)$ & $97(70 \%)$ & \\
\hline 3 & $74(29 \%)$ & $35(30 \%)$ & $39(28 \%)$ & \\
\hline 4 & $2(1 \%)$ & $1(1 \%)$ & $1(1 \%)$ & \\
\hline Computed tomography (CT) abdomen/pelvis & $255(100 \%)$ & $116(100 \%)$ & $139(100 \%)$ & n.s \\
\hline Diagnostic laparoscopy & $102(40 \%)$ & $43(37 \%)$ & $59(42 \%)$ & n.s \\
\hline Positron emission tomography (PET) scan & $49(19 \%)$ & $14(12 \%)$ & $35(25 \%)$ & 0.048 \\
\hline Endoscopic ultrasound (EUS) & $26(10 \%)$ & $5(4 \%)$ & $21(15 \%)$ & $\leq 0.001$ \\
\hline \multicolumn{5}{|l|}{ Overview } \\
\hline Direct to surgery & $202(79 \%)$ & $101(87 \%)$ & $101(73 \%)$ & $\leq 0.001$ \\
\hline Pre/post chemotherapy & $45(17 \%)$ & $9(8 \%)$ & $36(26 \%)$ & \\
\hline Post chemoradiation therapy (CXRT) & $8(3 \%)$ & $6(5 \%)$ & $2(1 \%)$ & \\
\hline
\end{tabular}

term surgical outcomes when stratified by extent of lymph node dissection (Table 3). Those undergoing a D2 resection were more likely to receive neoadjuvant chemotherapy, with $39(27 \%)$ patients compared to $6(6 \%)$ of the D1/D1+ group. The nodal count was significantly higher in the D2 resection group, with a median lymph node harvest of 24; the median lymph node harvest in the D1/D1+ resection group was 15 nodes. There were no significant differences with regard to operation type, blood loss, or overall morbidity rate between the two lymph node dissection groups. The median operating time in the D2 group was longer, at 210 versus $195 \mathrm{~min}$ in the D1/D1+ group $(p=0.006)$. The morbidity rate in the $\mathrm{D} 2$ resection group was $42 \%$ compared to $35 \%$ in the D1/D1+ group; this was not significant $(p=0.30)$. Anastomotic leakage was not significantly elevated in the D2 group $(p=0.27)$. There were four procedure-related deaths in the D2 group compared to none in the D1/D1+ group, also not statistically significant $(p=0.14)$. There was no statistically significant difference with regard to $\mathrm{T}$ stage or $\mathrm{N}$ stage between the two groups. There was a trend toward a higher stage within the D2 group, with $36 \%$ of cases being stage III or greater compared to $28 \%$ in the D1/D1+ group. There was no observed difference in overall survival between the lymph node dissection groups (Table 4).

\section{Discussion}

During the 12-year period studied, our unit managed an average of 43 gastric cancer patients per year with approximately 21 per year being suitable for curative resection shared among four surgeons. Compared with countries with larger populations and a higher incidence of gastric cancer, our results appear satisfactory although our center is defined essentially as a low-volume center. The overall operative mortality rate of $1.6 \%$ compares favorably with data from large contemporary audits, with the 2010 esophagogastric cancer audit of England and Welsh hospitals reporting an in-hospital mortality rate of $6 \%$ for patients undergoing curative gastric resection [31]. The operative mortality rate of $2.5 \%$ for our patients selected to have a D2 resection is comparable to the results published from internationally renowned centers [9, 17, 18, 20] and is significantly less than the mortality rates reported from two of the published randomized trials of D1 versus D2 resection [11, 32, 33]. It is worth noting that we have performed far fewer resections involving the pancreas and the spleen, which was a major source of morbidity in the Dutch study [11]. The pattern of morbidity we have reported is similar to other series involving "Western" patients [20], with a high incidence of cardiac and respiratory complications. 
Table 2 Surgical characteristics and perioperative outcomes of 255 curative gastric resections

\begin{tabular}{|c|c|c|c|c|}
\hline & All (255) & $2000-2005(n=116)$ & 2006-2011 $(n=139)$ & $p$ value \\
\hline Approach & & & & n.s \\
\hline Open & $234(92 \%)$ & $111(96 \%)$ & $123(89 \%)$ & \\
\hline Laparoscopic & $19(8 \%)$ & $5(4 \%)$ & $14(9 \%)$ & \\
\hline Laparoscopic converted & $2(1 \%)$ & 0 & $2(1 \%)$ & \\
\hline Operation & & & & n.s \\
\hline Total gastrectomy & $132(52 \%)$ & $65(56 \%)$ & $67(48 \%)$ & \\
\hline Subtotal gastrectomy & $123(48 \%)$ & $51(44 \%)$ & $72(52 \%)$ & \\
\hline Resection status & & & & n.s \\
\hline R0 & $245(96 \%)$ & $113(97 \%)$ & $132(95 \%)$ & \\
\hline $\mathrm{R} 1$ & $10(4 \%)$ & $3(3 \%)$ & $7(5 \%)$ & \\
\hline \multicolumn{5}{|l|}{ Nodal resection } \\
\hline D1 & $109(43 \%)$ & $86(74 \%)$ & $23(17 \%)$ & $\leq 0.001$ \\
\hline D2 & $146(57 \%)$ & $30(26 \%)$ & $116(84 \%)$ & \\
\hline \multicolumn{5}{|l|}{ Nodal counts } \\
\hline Nodes removed (median) & $21(0-77)$ & $16(2-75)$ & $24(0-77)$ & $\leq 0.001$ \\
\hline Nodes involved (median) & $1(0-41)$ & $1(0-35)$ & $1(0-41)$ & n.s \\
\hline Differentiation & & & & $\leq 0.001$ \\
\hline Well/moderate & $113(44 \%)$ & $68(59 \%)$ & $45(32 \%)$ & \\
\hline Poor/mucinous & $142(55 \%)$ & $48(41 \%)$ & $94(68 \%)$ & \\
\hline En bloc & & & & n.s \\
\hline Spleen & $11(4 \%)$ & $4(3 \%)$ & $7(5 \%)$ & \\
\hline Distal pancreas & $1(0.4 \%)$ & $1(1 \%)$ & 0 & \\
\hline Colon & $1(0.4 \%)$ & 0 & $1(1 \%)$ & \\
\hline Length of surgery (min) & $200(90-560)$ & $195(125-360)$ & $210(90-560)$ & 0.02 \\
\hline Blood loss (ml) & $200(0-2,800)$ & $200(0-1,300)$ & $200(0-2,800)$ & n.s \\
\hline Transfusion & $32(13 \%)$ & $13(11 \%)$ & $19(14 \%)$ & n.s \\
\hline Complications & $99(39 \%)$ & $40(35 \%)$ & $59(42 \%)$ & n.s \\
\hline Chest problems & $39(15 \%)$ & $17(16 \%)$ & $22(16 \%)$ & n.s \\
\hline Anastomotic leak & $23(9 \%)$ & $9(8 \%)$ & $14(10 \%)$ & n.s \\
\hline Wound infection & $12(5 \%)$ & $4(3 \%)$ & $8(6 \%)$ & n.s \\
\hline Cardiac problems & $19(7 \%)$ & $4(3 \%)$ & $15(11 \%)$ & 0.03 \\
\hline Length of stay (days) & $11(3-92)$ & $10(6-31)$ & $11(3-92)$ & 0.03 \\
\hline Operative mortality & $4(1.6 \%)$ & $1(1 \%)$ & $3(2 \%)$ & n.s \\
\hline Five-year overall survival & $53 \%$ & $52 \%$ & $57 \%$ & n.s \\
\hline
\end{tabular}

The reasons for our results are likely related to the surgery occurring in a unit that includes surgeons who have undergone training and education in gastric surgery and who also perform a large number of esophageal resections. Combined with this is the support received from experienced nursing staff, ready access to intensive care, and interventional radiology and also with a collaborative multidisciplinary oncology assessment of each patient. One group has examined the ability to handle adverse postoperative events and outcomes from gastric surgery, focusing on surgical volume [34]. The results demonstrated that the rate of complications was similar across all hospitals studied but the mortality rate was lower in the two higher- volume centers (defined as $>15$ resections per year) and the failure to rescue rate was significantly higher in lowervolume units compared to the two high-volume hospitals [34]. The ability to recognize adverse events early and to have access to the necessary nursing and medical support appears to be a key feature in improved outcomes for gastric cancer patients.

There are data demonstrating improved outcomes with increasing surgical volume for gastric resection. Birkmeyer et al. [27] observed the relationship between hospital volume and mortality across 14 operations; specifically, with gastric resection the hospitals that performed fewer than 5 resections annually had a mortality rate of $13 \%$ compared 
Table 3 Comparisons of outcomes stratified by lymph node dissection

\begin{tabular}{|c|c|c|c|}
\hline & $\mathrm{D} 1 / \mathrm{D} 1+$ & D2 & $p$ value \\
\hline Number of patients & 109 & 146 & \\
\hline Age (median) & 70 & 69 & n.s \\
\hline $\operatorname{Sex}(M: F)$ & $3: 2$ & $3: 2$ & n.s \\
\hline BMI (median) & 25 & 26 & n.s \\
\hline Treatment overview & & & $\leq 0.001$ \\
\hline Direct to surgery & $101(93 \%)$ & $101(69 \%)$ & \\
\hline Pre/post chemotherapy & $6(6 \%)$ & $39(27 \%)$ & \\
\hline Post CXRT & $2(2 \%)$ & $6(4 \%)$ & \\
\hline Nodal count (median) & $15(0-64)$ & $24(3-77)$ & $\leq 0.001$ \\
\hline Nodes involved (median) & $0(0-28)$ & $1(0-41)$ & n.s \\
\hline T stage & & & n.s \\
\hline $\mathrm{T} 1 / \mathrm{T} 2$ & $60(55 \%)$ & $68(47 \%)$ & \\
\hline $\mathrm{T} 3 / \mathrm{T} 4$ & $49(45 \%)$ & $78(53 \%)$ & \\
\hline Nodal status & & & n.s \\
\hline Node negative & $55(51 \%)$ & $60(41 \%)$ & \\
\hline Node positive & $54(50 \%)$ & $86(59 \%)$ & \\
\hline Postoperative stage & & & n.s \\
\hline IA & $23(21 \%)$ & $26(18 \%)$ & \\
\hline IB & $20(18 \%)$ & $27(19 \%)$ & \\
\hline IIA & $21(19 \%)$ & $18(12 \%)$ & \\
\hline IIB & $16(15 \%)$ & $21(14 \%)$ & \\
\hline IIIA & $12(11 \%)$ & $21(14 \%)$ & \\
\hline IIIB & $14(13 \%)$ & $27(19 \%)$ & \\
\hline IIIC & $1(1 \%)$ & $4(3 \%)$ & \\
\hline IV & $2(2 \%)$ & $2(1 \%)$ & \\
\hline Approach & & & n.s \\
\hline Open & $101(93 \%)$ & $133(91 \%)$ & \\
\hline Laparoscopy & $7(6 \%)$ & $12(8 \%)$ & \\
\hline Laparoscopy converted & $1(1 \%)$ & $1(1 \%)$ & \\
\hline \multicolumn{4}{|l|}{ Operation } \\
\hline Total gastrectomy & $53(49 \%)$ & $79(54 \%)$ & n.s \\
\hline Subtotal gastrectomy & $56(51 \%)$ & $67(46 \%)$ & \\
\hline Length of surgery (min) & $195(120-360)$ & $210(90-560)$ & 0.006 \\
\hline Blood loss (ml) & $200(0-2,800)$ & $200(0-1,300)$ & n.s \\
\hline Transfusion & $13(12 \%)$ & $19(13 \%)$ & n.s \\
\hline Complications & $38(35 \%)$ & $61(42 \%)$ & n.s \\
\hline Chest problems & $13(12 \%)$ & $26(18 \%)$ & n.s \\
\hline Anastomotic leak & $7(6 \%)$ & $16(11 \%)$ & n.s \\
\hline Wound infection & $4(4 \%)$ & $8(5 \%)$ & n.s \\
\hline Cardiac problems & $7(6 \%)$ & $12(8 \%)$ & n.s \\
\hline Length of stay (days) & $10(4-92)$ & $12(3-58)$ & n.s \\
\hline Operative mortality & 0 & $4(3 \%)$ & n.s \\
\hline Five-year overall survival & $58 \%$ & $49 \%$ & n.s \\
\hline
\end{tabular}

with hospitals performing $>19$ resections which had a mortality rate of $8.7 \%$. A large cohort study from the UK that analyzed 731 gastric cancer patients across 23 hospitals demonstrated that increasing surgical volume was associated with better staging and a reduction in mortality rate [28]. The 30-day mortality rate for the entire gastric cancer cohort was $14 \%$, and increasing surgical volume of both surgeon and hospital was inversely related to mortality. What constitutes a high-volume versus a low-volume center varies widely in reported series. The UK guidelines suggest a surgeon should be involved in 20 esophageal or gastric resections per year [35]. In Denmark, 20 cases per hospital is used as a marker of a high-volume center [36]. North American series have reported 14 or 15 gastric cancer cases per year as a marker of a high-volume gastric cancer center [27, 34]. In Asia, 200 cases per year are considered a marker of a high-volume institution [37-39]. Gastric cancer surgery has been centralized in some countries such as the UK, Denmark, The Netherlands, Sweden, and Finland [40]. In Denmark the impact of centralizing gastric cancer services from 37 hospitals to four to five units has led to a reduction in operative mortality rate from 8.2 to $2.4 \%$ [36] The incidence of gastric cancer is falling in Australia, so combined with our smaller population density we will never achieve the numbers of some of the large high-volume centers in other countries. The concentration of gastric cancer services in European countries has seen improvements in outcomes; whether this approach is applicable to our local conditions and geography is worthy of investigation.

Perhaps of equal significance is the evidence from these reports of the importance of those involved in gastric cancer utilizing MDT discussions of gastric cancer patients and being involved in thorough audits and review of surgical outcomes. During the 12 years studied we saw a difference in the management of these patients between the two 6-year time periods. There was an increase in the use of perioperative chemotherapy, from 8 to $26 \%$. Publication of the results of the MAGIC trial [22] in 2006 is the explanation for the rise in use of neoadjuvant chemotherapy. The results and application of this trial were readily implemented in our unit as an MDT with involvement of the oncology unit was already established. Additionally, we have increased the number of patients having a D2 lymph node dissection from 26 to $84 \%$ of cases. We have made these two changes without significantly increasing the operative morbidity or mortality in our group of patients. It is accepted that we currently apply a selective approach to lymph node dissection, with those undergoing a D2 resection being slightly younger and more likely to have undergone neoadjuvant therapy. A D2 lymph node dissection is more technically demanding and requires training and experience in the technique to perform it safely. However, with greater experience and with more confidence in performing the procedure we have increased our selection criteria in favor of a D2 resection. There are other limitations to this study: the database has 
Table 4 Five-year overall survival by stage (AJCC 6th edition)

\begin{tabular}{llllll}
\hline & SEER $(n=32531)$ & MSKCC $(n=1038)$ & NCC $(n=6730)$ & SNUH $(n=12026)$ & PAH $(n=255)$ \\
\hline Lymphadenectomy & D0, D1 & D2 D D1 & $>$ D2 & $>$ D2 & D2 $>$ D1 \\
Median nodes & $10-11$ & 22 & $>30$ & $>30$ & 21 \\
STAGE AJCC 6th edition & & & & \\
IA & 78 & 92 & 91.5 & 94.4 & 82 \\
IB & 58 & 85 & 84.6 & 84.2 & 64 \\
II & 34 & 49 & 69.3 & 68.5 & 67 \\
IIIA & 20 & 32 & 50.4 & 47.3 & 34 \\
IIIB & 8 & 11 & 30.6 & 31.6 & 20 \\
IV & 7 & 11 & 5.4 & 17.2 & 12 \\
\hline
\end{tabular}

SEER States Surveillance Epidemiology and End Results Database, USA; MSKCC Memorial Sloan Kettering Cancer Centre, New York, USA; NCC National Cancer Centre, Tokyo, Japan; SNUH Seoul National University Hospital, Seoul, South Korea; PAH Princess Alexandra Hospital, Brisbane, Australia [41]

prospectively maintained since 2005 but the data preceding this year have been retrospectively added, which introduces possible bias in comparing the two time periods. Currently, our morbidity data collect all events without using a staging system such as the Claviden-Dindo system, which may overrepresent minor morbidity and limit comparison with other studies. This system has been changed recently for all our new prospective data collection.

Our 5-year overall survival rate of $53 \%$ compares favorably with other series from Western centers. Table 4 compares our results with those published in a review by Schmidt and Yoon [41], who explored the role of lymphadenectomy in gastric cancer, illustrating that our overall survival when stratified by stage is comparable to rates from leading Western centers. The results from specialist centers in Asia are still superior to our current results; these differences are explored elsewhere [41]. Compared to earlier studies comparing survival with regard to D1 or D2 lymph node dissection, our survival figures are acceptable. Our D2 group had a 5-year overall survival rate of $49 \%$, which was less than that seen in our D1 group of $58 \%$. The bias in selection and the significant difference in the use of neoadjuvant chemotherapy in the D2 group compared to the D1/D1+ group limits the value of any direct comparison to our D1 group.

In conclusion, we have reported our outcomes in managing gastric cancer patients from a single upper GI unit during an 11-year period, with a R0 resection rate of $96 \%$, postoperative mortality rate of $1.6 \%$, postoperative morbidity rate of $39 \%$, and 5-year overall survival of $53 \%$. These data are comparable with leading international centers. During the 12-year period there has been an increase in the use of neoadjuvant therapy and D2 lymph node dissection. We have demonstrated that, despite being a relatively low-volume center by some definitions, with an average of 21 gastric resections annually, we can obtain these results with a combination of excellent perioperative support, experienced upper GI surgeons, MDT base patient management, and regular review of surgical outcomes. We assert that these features are necessary for those involved in the care of patients with gastric cancer.

\section{References}

1. Pisani P, Parkin DM, Bray F, Ferlay J. Estimates of the worldwide mortality from 25 cancers in 1990. Int $\mathrm{J}$ Cancer. 1999;83(1):18-29.

2. Jemal A, Siegel R, Ward E, Murray T, Xu J, Smigal C, et al. Cancer statistics, 2006. CA Cancer J Clin. 2006;56(2):106-30.

3. AIHW (Australian Institute of Health and Welfare) \& AACR (Australasian Association of Cancer Registries). Cancer in Australia: an overview, 2008. Cancer series no 46. Cat no. CAN 42. 2008.

4. Coburn NG. Lymph nodes and gastric cancer. J Surg Oncol. 2009;99(4):199-206.

5. Maduekwe UN, Yoon SS. An evidence-based review of the surgical treatment of gastric adenocarcinoma. J Gastrointest Surg. 2011;15(5):730-41.

6. Edge SB, Byrd DR, Compton CC, et al., editors. AJCC cancer staging manual. 7th edn. New York: Springer; 2009.

7. Sano T, Aiko T. New Japanese classifications and treatment guidelines for gastric cancer: revision concepts and major revised points. Gastric Cancer. 2011;14(2):97-100.

8. Japanese classification of gastric carcinoma, 3rd English edition. Gastric Cancer. 2011;14(2):101-12.

9. Sasako M, Sano T, Yamamoto S, Kurokawa Y, Nashimoto A, Kurita A, et al. D2 lymphadenectomy alone or with para-aortic nodal dissection for gastric cancer. $N$ Engl $J$ Med. 2008;359(5):453-62.

10. Sano T, Sasako M, Yamamoto S, Nashimoto A, Kurita A, Hiratsuka M, et al. Gastric cancer surgery: morbidity and mortality results from a prospective randomized controlled trial comparing D2 and extended para-aortic lymphadenectomy. Japan Clinical Oncology Group study 9501. J Clin Oncol. 2004;22(14):2767-73.

11. Bonenkamp JJ, Songun I, Hermans J, Sasako M, Welvaart K, Plukker JT, et al. Randomised comparison of morbidity after D1 
and D2 dissection for gastric cancer in 996 Dutch patients. Lancet. 1995;345(8952):745-8.

12. Bonenkamp JJ, Hermans J, Sasako M, van de Velde CJ, Welvaart $\mathrm{K}$, Songun I, et al. Extended lymph-node dissection for gastric cancer. N Engl J Med. 1999;340(12):908-14.

13. Tanizawa $Y$, Terashima M. Lymph node dissection in the resection of gastric cancer: review of existing evidence. Gastric Cancer. 2010;13(3):137-48.

14. Maruyama K, Sasako M, Kinoshita T, Sano T, Katai H, Okajima K. Pancreas-preserving total gastrectomy for proximal gastric cancer. World J Surg. 1995;19(4):532-6.

15. Biffi R, Chiappa A, Luca F, Pozzi S, Lo Faso F, Cenciarelli S, et al. Extended lymph node dissection without routine splenopancreatectomy for treatment of gastric cancer: low morbidity and mortality rates in a single center series of 250 patients. J Surg Oncol. 2006;93(5):394-400.

16. Kitamura K, Nishida S, Ichikawa D, Taniguchi H, Hagiwara A, Yamaguchi $\mathrm{T}$, et al. No survival benefit from combined pancreaticosplenectomy and total gastrectomy for gastric cancer. $\mathrm{Br} \mathbf{J}$ Surg. 1999;86(1):119-22.

17. Degiuli M, Sasako M, Ponti A. Morbidity and mortality in the Italian Gastric Cancer Study Group randomized clinical trial of D1 versus D2 resection for gastric cancer. $\mathrm{Br}$ J Surg. 2010;97(5):643-9.

18. Park CH, Song KY, Kim SN. Treatment results for gastric cancer surgery: 12 years' experience at a single institute in Korea. Eur J Surg Oncol (EJSO). 2008;34(1):36-41.

19. Siewert JR, Bottcher K, Stein HJ, Roder JD. Relevant prognostic factors in gastric cancer: ten-year results of the German Gastric Cancer Study. Ann Surg. 1998;228(4):449-61.

20. Strong VE, Song KY, Park CH, Jacks LM, Gonen M, Shah M, et al. Comparison of gastric cancer survival following R0 resection in the United States and Korea using an internationally validated nomogram. Ann Surg. 2010;251(4):640-6.

21. Smith JW, Shiu MH, Kelsey L, Brennan MF. Morbidity of radical lymphadenectomy in the curative resection of gastric carcinoma. Arch Surg. 1991;126(12):1469-73.

22. Cunningham D, Allum WH, Stenning SP, Thompson JN, Van de Velde CJ, Nicolson M, et al. Perioperative chemotherapy versus surgery alone for resectable gastroesophageal cancer. $\mathrm{N}$ Engl $\mathbf{J}$ Med. 2006;355(1):11-20.

23. Macdonald JS, Smalley SR, Benedetti J, Hundahl SA, Estes NC, Stemmermann GN, et al. Chemoradiotherapy after surgery compared with surgery alone for adenocarcinoma of the stomach or gastroesophageal junction. $\mathrm{N}$ Engl J Med. 2001;345(10):725-30.

24. Sakuramoto S, Sasako M, Yamaguchi T, Kinoshita T, Fujii M, Nashimoto A, et al. Adjuvant chemotherapy for gastric cancer with S-1, an oral fluoropyrimidine. $\mathrm{N}$ Engl $\mathrm{J}$ Med. 2007;357(18):1810-20.

25. Bang YJ, Kim YW, Yang HK, Chung HC, Park YK, Lee KH, et al. Adjuvant capecitabine and oxaliplatin for gastric cancer after D2 gastrectomy (CLASSIC): a phase 3 open-label, randomised controlled trial. Lancet. 2012;379(9813):315-21.
26. Begg CB, Cramer LD, Hoskins WJ, Brennan MF. Impact of hospital volume on operative mortality for major cancer surgery. JAMA. 1998;280(20):1747-51.

27. Birkmeyer JD, Siewers AE, Finlayson EV, Stukel TA, Lucas FL, Batista I, et al. Hospital volume and surgical mortality in the United States. N Engl J Med. 2002;346(15):1128-37.

28. Bachmann MO, Alderson D, Edwards D, Wotton S, Bedford C, Peters TJ, et al. Cohort study in South and West England of the influence of specialization on the management and outcome of patients with oesophageal and gastric cancers. $\mathrm{Br} \mathrm{J}$ Surg. 2002;89(7):914-22.

29. Gruen RL, Pitt V, Green S, Parkhill A, Campbell D, Jolley D. The effect of provider case volume on cancer mortality: systematic review and meta-analysis. CA Cancer $\mathrm{J}$ Clin. 2009;59(3):192-211.

30. Japanese gastric cancer treatment guidelines 2010 (ver. 3). Gastric Cancer 2011;14(2):113-123.

31. Palser T, Cromwell D, van der Meulen J, Hardwick R, Riley S, Greenaway K, Dean S. National Oesophago-Gastric Cancer Audit 2010. 2010.

32. Cuschieri A, Weeden S, Fielding J, Bancewicz J, Craven J, Joypaul V, et al. Patient survival after D1 and D2 resections for gastric cancer: long-term results of the MRC randomized surgical trial. Surgical Cooperative Group. Br J Cancer. 1999;79(9-10):1522-30.

33. Cuschieri A, Fayers P, Fielding J, Craven J, Bancewicz J, Joypaul $\mathrm{V}$, et al. Postoperative morbidity and mortality after D1 and D2 resections for gastric cancer: preliminary results of the MRC randomised controlled surgical trial. The Surgical Cooperative Group. Lancet. 1996;347(9007):995-9.

34. Smith DL, Elting LS, Learn PA, Raut CP, Mansfield PF. Factors influencing the volume-outcome relationship in gastrectomies: a population-based study. Ann Surg Oncol. 2007;14(6):1846-52.

35. Allum WH, Blazeby JM, Griffin SM, Cunningham D, Jankowski JA, Wong R. Guidelines for the management of oesophageal and gastric cancer. Gut. 2011;60(11):1449-72.

36. Jensen LS, Nielsen H, Mortensen PB, Pilegaard HK, Johnsen SP. Enforcing centralization for gastric cancer in Denmark. Eur J Surg Oncol. 2010;36(suppl 1):S50-4.

37. Nomura E, Tsukuma H, Ajiki W, Oshima A. Population-based study of relationship between hospital surgical volume and 5-year survival of stomach cancer patients in Osaka, Japan. Cancer Sci. 2003;94(11):998-1002.

38. Ahn HS, Yook JH, Park CH, Park YK, Yu W, Lee MS, et al. General perioperative management of gastric cancer patients at high-volume centers. Gastric Cancer. 2011;14(2):178-82.

39. Ioka A, Tsukuma H, Ajiki W, Oshima A. Hospital procedure volume and survival of cancer patients in Osaka, Japan: a population-based study with latest cases. Jpn J Clin Oncol. 2007;37(7):544-53.

40. Dikken JL, Verheij M, Cats A, Jansen EP, Hartgrink HH, van de Velde CJ. Extended lymph node dissection for gastric cancer from a European perspective. Gastric Cancer. 2011;14(4):396-8.

41. Schmidt B, Yoon SS. D1 versus D2 lymphadenectomy for gastric cancer. J Surg Oncol. 2013;107:259-64. 